\title{
Le Ps 18 Réaffirmation Messianique Dans le Cadre du Psautier en Relation à 1-2 Samuel et Divers Passages Bibliques
}

\author{
BERNARD GOSSE
}

\begin{abstract}
The Messianic reaffirmation ${ }^{1}$ of Ps 18 must be understood in the context of the influence of the book of Proverbs, more specifically the influence of Prov 30:1-14, upon the Psalter. However, it is not only the influence of Proverbs that is important. It goes further; for example; the psalm also responds to the denunciation of the fate of the poor at the return from the exile. These responses indicate some relations to late redactions of the Book of the Twelve, Jonah, Zephaniah (see Zechariah), Habakkuk, the Prayer of Hannah in 1 Sam 2:1-10 (which frames the books of Samuel together with 2 Sam $22=P s$ 18), or from the book of Job and some passages depending on Judges 5:4, later found in Ps 68, Dt 33:2 or Is 34 and 63:1-6.

RÉSUMÉ
\end{abstract}

Le Ps 18 doit se comprendre dans le contexte de l'influence du livre des Proverbes et plus spécialement Pr 30,1-14 sur le Psautier. Toutefois le Ps 18 ne se contente pas de prendre en compte des passages des Proverbes, il réagit en apportant des réponses aux dénonciations $d u$ sort du pauvre marginal au retour de l'exil. Ces réponses témoignent de relations avec des rédactions tardives du Livre des Douze, Jonas, Sophonie (voir Zacharias), Habaquq, du cantique de Anne en 1 Sam 2,1-10 (qui encadre les livres des Samuel avec 2 Sam $22=$ Ps 18) ou du livre de Job et des reprises du thème de Jug 5,4, dans le Ps 68, Dt 33,2 ou Is 34 et 63,1-6.

* Submitted: 28/07/2020; peer-reviewed: 16/10/2020; accepted: 12/11/2020. Bernard Gosse, "Le Ps 18 réaffirmation messianique dans le cadre du Psautier en relation à 1-2 Samuel et divers passages bibliques," Old Testament Essays 33 no. 3 (2020): 582-595. DOI: https://doi.org/10.17159/2312-3621/2020/v33n3a12.

1 Il s'agit d'une réaffirmation non seulement du Messie David mais également sa descendance comme en témoigne Ps 18,51. Ce second point est crucial après l'exil. 
KEYWORDS: Psalter, Proverbs 30:1-14, Psalm 18:1-2 Samuel, The Book of the Twelve, Messianic, The Poor

\section{A INTRODUCTION}

Le titre de Ps 18,1, fait référence aux récits des persécutions subies par David dans les livres de Samuel, ${ }^{2}$ dans la continuité de l'usage par le Psautier de titres davidiques longs dans le second psautier davidique Ps 51-72, titres s'appuyant sur des passages des livres de Samuel où David est persécuté, par accrochage verbal avec le contenu du corps du psaume, en Ps 51,1-2; 52,1-2 ; 54,1-2 ; 56,1; 59,$1 ; 60,1-2 .{ }^{3}$ Le titre de Ps 18,1 , se présente comme une réponse aux titres anxiogènes quant au sort de David, du second psautier davidique, particulièrement avec la mention de Saül. ${ }^{4}$ On peut noter que Saül (שאול) est mentionné dans le Psautier uniquement dans les titres des Ps $18 ; 52 ; 54 ; 57$; 59 , comme représentant une menace de mort pour David, à la manière du Shéol (שאול) ; alors que le Shéol est mentionné 16 fois dans le corps des psaumes, dont 18,6 , ce qui souligne un enracinement beaucoup plus profond dans la formation des psaumes. Dans les livres de Samuel nous avons, au contraire, seulement deux attestations du Shéol en 1 Sam 2,6 (Cantique d'Anne, 1 Sam 2,1-10) et 2 Sam 22,6 (//Ps 18) qui encadrent les mentions de Saül dans de nombreux passages des livres de Samuel, particulièrement les récits des persécutions de David par Saül. Le processus d'identification de Saül avec le Shéol, comme menace de mort pour David, apparait donc clairement avec les titres des psaumes en lien avec les cantiques de 2 Sam 22 et 1 Sam 2,1-10. Le Ps 18, peut alors pratiquer une réaffirmation messianique, de David et de sa descendance, et par là même

2 Le caractère royal du psaume est souligné par le parallèle de 2 Sam 22. Voir Nancy Declaissé-Walford, Rolf A. Jacobson et Beth Laneel Tanner, The Book of Psalms (Cambridge: Eerdmans, 2014), 191.

3 Les titres davidiques longs du second psautier davidique Ps 51-72, renvoient à des passages particuliers des livres de Samuel. Le titre du Ps 18 constitue un renvoi beaucoup plus large à la rédaction des livres de Samuel. Il apporte une réponse globale et non plus ponctuelle, vis-à-vis d'un événement particulier. Bernard Gosse, "L'insertion de 2 Sam 22 dans les livres de Samuel, et l'influence en retour sur les titres davidiques du Psautier," JANES 27 (2000) : 31-47.

4 En Ps 18,1, David est délivré de tous ses ennemis, dont particulièrement Saül son principal ennemi. Voir dans les titres les mentions de Ps 52 : Saül ; Ps 54 : Saül : Ps 55 : Les Philistins; Ps 57: Saül ; Ps 59 : Saül; Ps 60: Aram et Edom. On peut y ajouter Ps 3,1, qui imite les titres du second psautier davidique, avec la mention de la menace d'Absalom. Le rapprochement opéré par le titre du Ps 3 avec le passage des livres de Samuel concernant la fuite devant Absalom, est encore plus problématique et moins vraisemblable que dans le cas des titres du second psautier davidique. Mais d'un point de vue rédactionnel, il prépare, dès l'ouverture du premier psautier davidique Ps 3-41, l'annonce de la libération devant tous les ennemis du Ps 18. Dans le Psautier les titres des psaumes jouent un rôle rédactionnel important au niveau de la constitution de collections de psaumes et finalement de l'ensemble du livre. 
également de Yahvé, dont David a fait monter l'arche à Jérusalem. ${ }^{5}$ Il s'agit d'une réponse aux inquiétudes du psautier élohiste, ${ }^{6}$ quant au sort de la dynastie, mais également aux inquiétudes du livre des Proverbes quant au pauvre, marginal postexilique. Sur ce dernier point en effet, le Ps 18 prend également en compte la situation du pauvre du retour de l'exil, telle qu'elle est décrite dans le livre des Proverbes ainsi que dans le premier psautier davidique Ps 3-41, qui en dépend sur ce thème. Car, de même qu'il apporte une réponse positive au sort de David et sa descendance, le Ps 18 répond également en faveur des pauvres, suite aux dénonciations des exactions contre ces derniers par le livre des Proverbes. ${ }^{7}$ Nous allons voir que le Ps 18 , entretient également des relations littéraires avec divers passages généralement tardifs du livre des Proverbes, audelà du cas de Pr 30,1-14, ainsi qu'avec les reprises de Nb 10,35 et Jug 5,4-5 dans diverses rédactions dont le Ps 68 qui a influencé le premier psautier davidique Ps 3-41, quant à la défense du pauvre marginal postexilique. Le Ps 18 doit également être compris en relation au cantique d'Anne en 1 Sam 2,1-10, ${ }^{8}$ qui dans le cadre des livres de Samuel, opère une réaffirmation messianique en inclusion avec 2 Sam 22, texte parallèle du Ps 18. Par ailleurs le Ps 18 présente des points communs avec le livre de Jonas quant au thème de la menace du Shéol identifié à la menace des grandes eaux, et a connu des relectures dans les livres de Job, Sophonie, Zacharie et Habaquq.

\section{B LA RÉAFFIRMATION MESSIANIQUE ET DU PAUVRE, DANS LE CADRE DU PS 18 SELON PR 30,1-14, EN RELATION AUX PERSÉCUTIONS SUBIES PAR DAVID SELON LES TITRES DU PSAUTIER ET LES LIVRES DE SAMUEL}

Le texte de Ps 18,3: "Yahvé est mon roc (סלעי) et ma forteresse (ומצודתי), mon libérateur (ומפלטי), c'est mon Dieu. Je m'abrite (מניטה) (צחהי) (משרי), mon bouclier (מגני) et ma force (מקרן) de salut (ישעי), ma citadelle (משגבי)", applique à David les perspectives de Ps 18,31 : "Dieu, sa voie est sans reproche et la parole (צמרת) de Yahvé (צרופה)) est éprouvée (מרוה). Il est, lui, le bouclier (מגן) de quiconque s'abrite (החסים) en lui (בוה).". Ce dernier passage est conforme

5 Dans le Psautier le terme ארון n'apparaît qu'en Ps 132,8, en relation avec un pèlerinage sur les pas de David, qui y a fait monter l'arche. Mais ארון est très fréquent dans les livres de Samuel auxquels appartiennent 2 Sam 22. Et surtout l'arche ארון, joue un rôle majeur en 1 Ch 15-16, en référence à David faisant monter l'arche à Jérusalem, et aux rôles de chantres lévites des Asaphites et des Ezrahites.

6 Inquiétude quant au sort de la dynastie davidique, dont le sort est étroitement lié à la montée de l'arche de Yahvé à Jérusalem par David.

7 Bernard Gosse, "La réaffirmation messianique du Ps 18 dans la perspective du salut du marginal au retour de 1'exil, " ZAW 130 (2018): 586-601.

8 Beat Weber, Werkbuch Psalmen I. Die Psalmen 1 bis 72 (Stuttgart: Verlag W. Kohlhammer, 2001), 108. " (vgl. auch 28.51 mit 1. Sam 2,4.7.10, sowie 29 mit 2. Sam 21,17)." 
à l'influence de Pr 30,5: "Toute parole (מרון) (מרוה) de Dieu est éprouvée (צרופה), il est un bouclier (מגן) pour qui s'abrite (לחסים) en lui (בור)." ${ }^{9}$ (10 Les autres termes de Ps 18,3, se référant à une "protection" de David, sont empruntés à d'autres passages, de psaumes à titres davidiques, ou des livres de Samuel faisant allusions à la menace de Saül ou autre persécuteur. Pour commencer, dans un premier temps, au niveau des titres du Psautier, étant donné que le titre du Ps 18 répond aux titres anxiogènes du second psautier davidique, dont celui du Ps 59 (où Saül veut mettre David à mort), on peut rapprocher le terme משגב de Ps 18,3 des emplois de Ps 59,10.17.18. ${ }^{11}$ Dans un deuxième temps, la mention de Saül (שאול) en Ps 18,1, permet d'établir un lien avec les livres de Samuel, à partir d'une relecture du terme Shéol (שאול) ${ }^{12}$ de Ps 18,6. Avec le titre du Ps 18, la menace du Shéol est réinterprétée en fonction de la menace que représente Saül dans les livres de Samuel. Dans ce contexte, le reste du vocabulaire de Ps 18,3, correspond à la prise en compte de passages des livres de Samuel où David est en difficulté, particulièrement les récits de 1 Sam 22-24 où David est persécuté par Saül, comme סלע : 1 Sam 23,25.28, ${ }^{13}$ en référence à un lieu de refuge de David. Il en est de même pour מצודה : Sam 22,4.5; 1 S4,23; terme appliqué à Jérusalem conquise par David en 2 Sam 5,7.9. Le verbe פלר : פל כerrespond au terme פליטה de 2 Sam 15,14, au sujet de la fuite de David et ses serviteurs devant Absalom cette fois. ${ }^{14}$ Le terme צור peut faire référence à 1 Sam 24,3, et à l'épisode de la grotte qui suit, mais en même temps il opère un renvoi au même terme en 1 Sam 2,2, de même que קרן, Ps 18,3 (2 Sam 22,3) et 1 Sam 2,1 dans

9 Le verbe חס, au participe poël, apparaît dans la Bible, en Pr 14,32 ; 30,5 ; Ps 2,12 ; 5,$12 ; 17,7 ; 18,31(=2$ Sam 22,31$) ; 31,20 ; 34,23$; et en Is 57,13 et Nah 1,7 . Ce phénomène se comprend bien dans le cadre de l'influence de $\operatorname{Pr} 30,5$, sur le premier livre du Psautier. A d'autres formes verbales, le verbe חסה, apparaît encore 9 fois en Ps 3-41, pour 9 autres emplois dans le Psautier et 6 autres emplois bibliques.

10 J'ai déjà fait le rapprochement entre Ps 18,31-32 et 30,5.9, et ses conséquences pour la prise en compte du Ps 19. Bernard Gosse, "Le Ps 19 prolongement du Ps 18 : Baal, El, Yahvé, le triple parallélisme synonymique, l'influence du livre des Proverbes et la Loi et David", ZAW 129 (2017) : 568-582. Le plan du Ps 19 découle de Ps 18,31-32.

11 Selon de titre de Ps 59,1, Saül fait espionner la maison de David pour le mettre à mort. Le terme משגב n'apparaît pas dans les livres de Samuel. Il est présent presque exclusivement dans le Psautier au sujet de la protection divine, y compris en Ps 144,2, qui reprend largement le vocabulaire de Ps 18,3. (משגב: 13 emplois ; 10 fois dans le Psautier ; et en Is 25,12 et Jr 48,1 : la cité forte de Moab détruite ; l'emploi de Is 33,16 est à rapprocher de Ps 18,34, du Psautier et des Proverbes).

12 Terme (שאול) Shéol en 1-2 Samuel : 1 S 2,6; 2 S 22,6. Ces attestations encadrent les mentions de Saül dans les livres de Samuel, à partir de $1 \mathrm{~S} \mathrm{9,2,} \mathrm{une} \mathrm{multitude} \mathrm{de}$ fois, avec le dernier emploi en $2 \mathrm{~S} 22,1$. Ce point souligne les correspondances entre les deux cantiques, qui prennent les livres de Samuel en inclusion.

13 Voir Ps 54,2 et 1 S 23,19-28 (persécution de David par Saül).

14 Voir le titre du Ps 3, qui imite les titres anxiogènes du second psautier davidique, Ps 51-72. De ce point de vue le titre du Ps 3, prépare la réponse à ces titres dans le cadre du premier psautier davidique, particulièrement le Ps 18. 
une même perspective de réaffirmation davidique. D'autant plus que dans le cadre des livres de Samuel, le cantique de 1 Sam 2,1-10 fait inclusion avec 2 Sam 22, parallèle du Ps 18. Ce point souligne la réinterprétation des livres de Samuel, en termes de réaffirmation messianique davidique, par ces deux cantiques de manière conjuguée. On peut noter la même thématique en Ps 18,28 (2 Sam 22,28): "Toi qui sauves le peuple des humbles et rabaisse (תשפיל) les yeux hautains (רמות)" et 1 Sam 2,7b : "qui abaisse (משפיל) et aussi qui élève (מרומם)." Le Ps 18 (parallèle de 2 Sam 22) doit donc être lu dans le cadre des cantiques bibliques qui réinterprètent les livres qui se réfèrent à l'histoire d'Israël, ici les livres de Samuel. Ce point est souligné par l'usage, dans la Bible, du terme שירה : Ex 15,1;2 Sam 22,1; Ps 18,1; Is 5,1; 23,15;Am 8,3; Nb 21,17; Dt 31,19.19.21.22.30 (introduction au cantique de Dt 32) ; 32,44, pour toute la Bible. ${ }^{15}$ Le texte de Ps 18,11: "il chevaucha (כוירכב) un chérubin (כרוב) et vola, il plana sur les ailes du vent (רוח)," correspond également au fait que la mention des chérubins se comprend bien, en référence aux livres de Samuel, Yahvé siège sur les chérubins (הכרבים) en 1 Sam 4,4; ${ }^{16} 2$ Sam 6,2. Et l'arche est utilisée dans les batailles.

Ce contexte assure une continuité avec l'usage du Ps 68, dans le cadre du premier psautier davidique, qui s'appuie pour présenter les interventions de Yahvé, sur celles de $\mathrm{Nb} 10,35$ en Ps 68,2 et Jug 5,4-5 en Ps 68,8-9. ${ }^{17}$ Au sujet des chevauchées de Yahvé, on peut relever Ps 18,10: "Il inclina les cieux et descendit (וירד), une sombre nuée (וערפל) sous ses pieds", et Ps 68,5: "Chantez à Dieu, jouez pour son nom, frayez la route au Chevaucheur (לרכר ) des nuées (בערבות), son nom est Yahvé, exultez devant sa face."18

L'influence du livre des Proverbes sur le Psautier et spécialement ici le Ps 18 , ne se limite pas à l'usage de $\operatorname{Pr} 30,1-14$. On peut rappeler l'influence d'autres passages des livres des Proverbes sur le Ps 18. ${ }^{19}$ Ainsi le psalmiste, qui a été

15 Dans le Psautier c'est le terme שיר qui est utilisé fréquemment. C'est une indication que le Ps 18 doit plutôt être considéré comme un cantique comme son parallèle de 2 Sam 22, dans le cadre d'une réaffirmation messianique dans les livres de Samuel. La différence entre שירה (masculin) et שיר (féminin) est minime. Mais le caractère systématique et non aléatoire dans les titres du Psautier, semble bien indiquer une volonté de différenciation. Et dans le cas du Ps 18, c'est la forme féminine qui a été choisie, seul cas dans le Psautier.

16 En vue d'une intervention dans la bataille.

17 Bernard Gosse, "La réaffirmation messianique du Ps 18 dans la perspective du salut du marginal au retour de l'exil," ZAW 130 (2018): 588, Nb 10,35 et Ps 68,2; 592-593: Jug 5,4-5 et Ps 68,8-9.

18 Gosse, “La réaffirmation messianique du Ps 18," 595-598: “Application du salut du Ps 68 à David et au pauvre opprimé du premier psautier davidique, auquel David est identifié par les titres et particulièrement le Ps 18."

${ }^{19}$ Bernard Gosse, L'influence du livre des Proverbes sur les rédactions bibliques à l'époque Perse (Sup Trans 14; Paris: Gabalda, 2008), 62-63. 
identifié à David par le titre du psaume, proclame le jugement rendu en sa faveur en Ps 18,21: "Yahvé me rend (יגמלני) (כידי) (כצדקי), selon la pureté

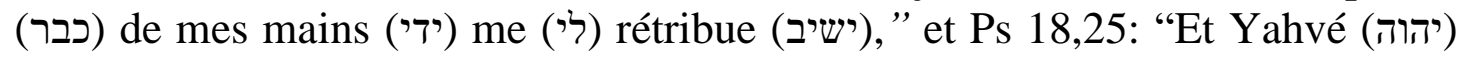

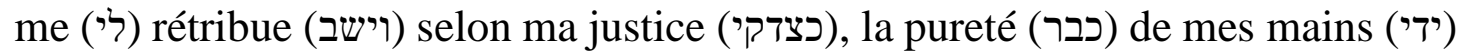
devant (לנגד) (ל) $^{21}$ ses yeux," conformément à Pr 24,12b: "Alors qu'il sait, lui qui t'observe; c'est lui qui rendra (להדיב) à l'homme selon son œuvre (כפעלו), ${ }^{22 ، “}$ on reconnaît en Ps 18,21.25 et Pr 24,12, la même construction, verbe שוב (Hiphil) + préposition ל ל préposition $3 .{ }^{23}$ Voir encore Pr 24,29b: "Je rendrai (אשיב) à chacun (לאיש) selon son œuvre (כפלוב)!"

L'usage du verbe חסד au hitpael en Ps 18,26: "26 Tu es fidèle (תתחסד) avec le fidèle (חסיד), sans reproche (תחדמם) avec l' (גבר) irréprochable (תמים)," répond à l'usage du même verbe חou piel en Pr 25,10: "de crainte que celui qui entend ne te bafoue (יחסדך) et que ta diffamation soit sans retour." Le verbe TOח, apparaît trois fois dans la Bible, d'une part au piel en Pr 25,10 et d'autre part au hitpael en 2 Sam 22,26 et Ps 18,26, avec un sens opposé à l'usage du verbe au piel en Pr 25,10. La volonté d'utiliser le verbe non Ps 18,26 au hithpael (avec un sens qui met en valeur l'opposition de signification, avec l'usage du verbe en Pr 25,10), ${ }^{24}$ est soulignée par l'usage de la même forme verbale du verbe תמם (hithpael): 2 Sam 22,26; Ps 18,26, pour toute la Bible. Des formes verbales rares sont construites à partir des substantifs correspondants.

En Ps 18,27: "pur (נבר) avec qui est pur ( עם (ופ-תתברר) mais rusant (תתפתל) avec le fourbe (ועם-עקש)", on retrouve un double usage du hithpael. On peut penser à une déclinaison du כבר ידי 'de Ps 18,21. La deuxième partie de 18,27 correspond à l'opposé de Pr 8,8: “(la Sagesse) Toutes les paroles de ma bouche

20 La première formulation est construite avec le verbe גמל, mais elle est retranscrite aussitôt avec la formule parallèle des Proverbes. La construction avec le verbe גמל, apparaît dans le Psautier et les livres de Samuel.

21 On peut noter l'usage répété des prépositions $כ$ et ל.

22 Le terme פעל n'apparaît dans les livres de Samuel qu'en 2 Sam 23,20. Or 2 Sam 23 présente des ressemblances avec Pr 30,1-3 ou 31,1-2, et pourquoi pas avec d'autres passages des livres des Proverbes? Le texte de 2 Sam 23, présente également des ressemblances avec $\mathrm{Nb} 24$, probablement en raison de la réaffirmation messianique de $\mathrm{Nb} 24,17$, ce qui rejoint les perspectives de 2 Sam 22 et Ps 18. Bernard GOSSE, "2 Samuel 23,1-7, en relation avec le Psautier, le Pentateuque et le livre de Malachie en vue de la réaffirmation de David et sa descendance en réponse à leur substitution par Moïse et en lien avec l'influence du livre des Proverbes sur les rédactions bibliques," OTE 31 (2018): 389-398.

23 La doctrine de la rétribution apparaît par ailleurs, Jb 34,11; Sir 16,12-14; Jr 32,19; Ps 62,13. Mais nous soulignons ici la construction grammaticale.

24 David J.A. Clines, ed., The Dictionary of Classical Hebrew Volume III ט-T (Sheffield: Sheffield Academic Press, 1996), 277, חסד I, Hithpael: "show oneself loyal; 2 Sam 22,26 // Ps 18,26) ;סד " חII, piel: " bring shame upon, reproach; Pr 25,10 ”. 
sont justes, en elles (בהם) rien (נפתל) ni de faux (ועקש)."25 Yahvé ruse avec le fourbe pour contrer son action. ${ }^{26}$

C'est justement cette discrimination, entre le pur et le fourbe, qui est exprimée en Ps 18,28 : "toi qui sauves (עושיע) le peuple des humbles (עם-עני), et rabaisses (תשפיל) les yeux (רמות) (רינים), hautains pour défendre les humbles par rapport aux orgueilleux en réponse à la situation décrite en $\operatorname{Pr} 30,13-14$ : "engeance aux regards (עיניו) altiers (רמו) et aux paupières hautaines, 14 engeance dont les dents sont des épées, les mâchoires des couteaux, pour dévorer les pauvres (עניים) et les retrancher du pays, et les malheureux, d'entre les hommes." 28

Suite au passage de Ps 18,31 qui reprend Pr 30,5, le rétablissement de la justice permet une réaffirmation théologique yahviste, en Ps 18,32 : "Qui (מי) donc est Dieu (אלוה), hors Yahvé (יהוה)? Qui est Rocher (צור), sinon notre Dieu?". Cette réaffirmation constitue une réponse aux incertitudes de $\operatorname{Pr} 30,9 \mathrm{a}$ : "de crainte que comblé, je ne me détourne et ne dise: "Qui (מיה) (יהוה)? Cette réaffirmation yahviste est à mettre en relation avec la réaffirmation davidique du Ps 18, c'est David qui a fait monter l'arche de Yahvé à Jérusalem et Salomon qui l'a installée dans le Temple. Le regain de la tradition yahviste, y compris dans le Pentateuque, est également à rapprocher de ce processus. ${ }^{29} \mathrm{En}$ Esd 1,2, Cyrus est présenté comme identifiant Yahvé au "Dieu du ciel." Ps 18,32, présente Yahvé comme le seul Dieu.

25 עקש, 7 emplois dans le livre des Proverbes, Ps 18,27; 2 Sam 22,27; Ps 101,4 (titre davidique et rejet des dévoyés...); Dt 32,5 (dénonciation d'une génération fourbe... dénuée de sagesse) pour toute la Bible.

26 Verbe פתל dans la Bible : Niphal: Pr 8,8; Job 5,13; Gn 30,8; Hithpael : 2 Sam 22,27; Ps 18,27. David J.A. Clines, ed., The Dictionary of Classical Hebrew, Volume VI 0 (Sheffield: Sheffield Phoenix Press, 2007): 811: פתל; Niphal b (Pr 8,8): "croaked thing, perverse thing"; Hithpael (Ps 18,27): "show oneself shrewd, show oneself crafty."

27 Voir l'anagramme entre עין

28 On retrouve la même thématique de Pr 30,13-14 en Ps 109,16, au sujet des pauvres et des malheureux. En Ps 109,17-18, nous relevons ensuite la dénonciation de l'usage de la malédiction au lieu de la bénédiction comme en Pr 30,11. Bernard Gosse : "L'influence de Pr 30,11-14 sur le Ps 109 dans la continuité de celle de Pr 30,1-14 sur le Ps 18 et la dénonciation de la pratique de la malédiction," ZAW 132 (2020): 415426. Dans la réaffirmation davidique des Ps 108-110, les Ps 108 et 110 concernent les ennemis extérieurs et le Ps 109 les ennemis intérieurs, les riches impies du retour de l'exil.

29 Bernard Gosse, “ La tradition yahviste en Gn 6,5-9,17,” HENOCH 15 (1993): 139_ 154. 
Dans le cadre de l'influence du Ps 68 sur le Ps $18,{ }^{30}$ Ps 18,39: “je les frappe (אמחצם), ils ne peuvent se relever, ils tombent, ils sont sous mes pieds

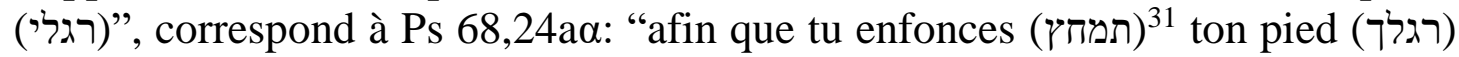
dans le sang", en lien avec la réaffirmation messianique en $\mathrm{Nb} 24,17$ et finalement Ps 110,5.6. ${ }^{32}$ Le texte de Ps 68.2 qui s'appuie déjà sur $\mathrm{Nb} 10,35$, en rapport à l'usage de l'arche dans les combats, pouvait s'inspirer de la réaffirmation messianique de $\mathrm{Nb} 24,17$ : "Je le vois - mais non pour maintenant, je l'aperçois - mais non de près. Un astre issu de Jacob devient chef, un sceptre se lève, issu d'Israël. Il frappe (ומחץ) les tempes de Moab et le crâne de tous les fils de Seth". ${ }^{33}$

\section{LE PS 18 DANS LA CONTINUITÉ DUPS 68, SES LIENS AVEC $1 \mathrm{~S}$ 2,1-10, LES RELATIONS AVEC LES LIVRES DE JOB, JONAS SOPHONIE ZACHARIE ET HABAQUQ}

Ps 68; Jug 5,4-5; Nb 10,35; 2 Sam 22; 1 Sam 2,1-10

Nous avons vu que dans le retournement en faveur du psalmiste identifié à David et également au pauvre postexilique, le Ps 18 , prenait en compte le Ps 68 , ce qui est vrai d'une manière beaucoup plus large au niveau du premier psautier davidique, avec la défense du pauvre en référence à l'intervention divine symbolisée par la présence de l'arche d'alliance. Or Jug 5,4-5 a influencé Ps 68,8-9 et $\mathrm{Nb} 10,35$ également Ps $68,2 .{ }^{34}$ Le Ps 18 est également construit en

30 Dans le cadre de l'influence du Ps 68 sur l'ensemble du premier psautier davidique. Yahvé se levant en faveur des pauvres, pour répondre aux dénonciations du livre des Proverbes.

31 En Ps 68,22, c'est Dieu le sujet du verbe מחצ.

32 מחץ: Ps 18,39; 68,22.24; 110,5.6, dans le Psautier; Nb 24,8.17 dans le livre des Nombres, toujours dans le cadre d'une réaffirmation messianique. Le Ps 110 participe de la réaffirmation messianique dans le cinquième livre du Psautier, en inclusion avec le premier livre. Mais le terme de Messie ne sera utilisé qu'en Ps 132,10.17, alors qu'il est présent en Ps 18,51.

$33 \mathrm{Nb} 24,17$ correspond à une réaffirmation messianique (située artificiellement dans un lointain passé, juste avant l'entrée en terre promise), dans la même ligne de réaffirmation que dans les Ps 18 et 110. Les oracles de Balaam qui précédent en $\mathrm{Nb} 22$ 24, traitent de la question de la malédiction et de la bénédiction, voir également Ps 109,28 et Pr 30,11. Le Ps 18 a lui-même été influencé par Pr 30,1-14. La question de la malédiction et de la bénédiction est encore présente dans la généalogie de Jésus (qui suppose en même temps la réaffirmation messianique) en Mat 1,1-14. Bernard Gosse, "The 42 Generations of the Genealogy of Jesus in Matt $1: 1-17$, and the Symbolism of Number 42, Curse or Blessing, in the Bible and in Egypt," Studia Biblica Slovaca 10 (2018): 142-151.

${ }^{34}$ Bernard Gosse, "La question du pauvre dans le premier psautier davidique, Ps 3-41, comme réponse à Pr 30,13-14”, OTE 32 (2019) : 548-551 : “D La référence à l'arche d'alliance dans le Ps 68 et son influence sur le premier psautier davidique ". Ps 68,2, s'appuie sur $\mathrm{Nb} 10,35$. 
interaction avec le cantique de 1 Sam 2,1-10, cantique qui encadre une relecture des livres de Samuel en inclusion avec 2 Sam 22 parallèle au Ps 18. Quant au thème primitif du Ps 18, celui du Shéol (שאול), avant la relecture par le titre de 18,1 en référence au roi Saül, il apparait avec la mention de la menace des eaux, comme ayant des parallèles dans le livre de Jonas.

\section{Jonas}

Le texte de Ps 18,5-7: "5 Les flots de la Mort m'enveloppaient, les torrents

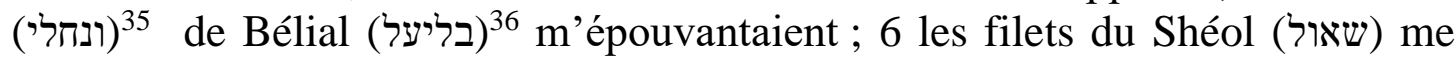

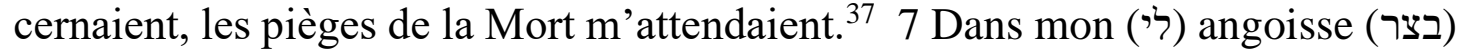
j'invoquai (ארא) Yahvé (יהוה), vers mon Dieu je lançais mon cri (אשוע), il

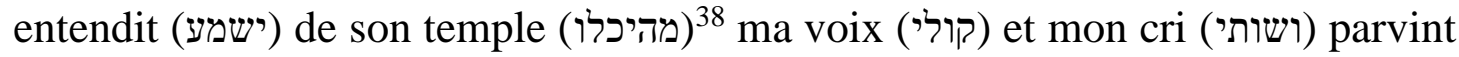
à ses oreilles", présente de nombreux parallèles, quant aux thèmes de l'angoisse devant les menaces du Shéol manifestées par des grandes eaux et l'appel par un cri à Yahvé, qui répond depuis son temple, avec Jonas 2,2-4: "2 Des entrailles du poisson, Jonas pria Yahvé (יהוה), son Dieu. 3 Il dit: J'invoquai (קרוה (מריה), (מריה), depuis

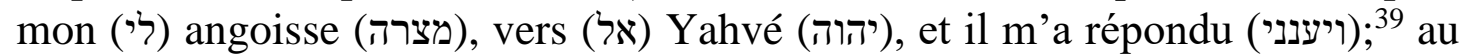
sein du shéol (שאול), je lançais mon cri (שועתי), tu as entendu (שמותי) ma voix (קולי). 4 Tu m'avais jeté dans les profondeurs, au cœur de la mer, et le flot (ונהר) m'environnait. Toutes tes vagues et tes lames ont passé sur moi. 5 Et moi je disais : Je suis rejeté de devant tes yeux. Pourtant je continue de regarder vers ton saint Temple (היכל)!"' Dans le livre de Jonas, la prière arrive jusqu'au saint Temple (היכל) en 2,8. Toutefois un élément particulier apparaît en Ps 18,5, la mention des "torrents de Bélial" (le thème des grandes eaux). Ce point semble lié à la relecture du Shéol en référence aux livres de Samuel, par le titre de Ps 18,1. En effet dans les livres de Samuel les gens de Bélial, apparaissent comme

\footnotetext{
${ }^{35}$ Alison Ruth Gray, Psalm 18 in Words and Pictures. A Reading Through Metaphor (Boston: Brill, 2014), 71." In the Psalms, water and waves function as powerful symbols of threat, danger, Chaos and enemies (e.g. Pss 32.6; 69.1) and are ever used to describe YHWH's own treatment of the psalmist (e.g. Ps 42.8, cf. Jon 2.4, 6)".

36 Graham Davies and Robert Gordon, Studies of the Language and Literature of the Bible. Selected Works of J.A. Emerton (Boston: Brill, 2015), 97-100: "Sheol and the Sons of Belial", p. 100 (au sujet de Belial): "While the view that it is a word for Sheol is not impossible, it is more probable that it means "destructiveness" or the like."

37 Hans-Johachim Kraus, Psalms 1-59 (Minneapolis: Augsburg Publishing House, 1988), 260: "שיעל and are the powers that rage in the sphere of ." Alison Ruth Gray, Psalm 18, 73. Voir Jon 2,3; Hab 2,5.

38 היכל: 1 S 1,9; 3,3 (Silo); 2 S 22,7 dans les livres de Samuel; Jonas 2,5.8.

39 Verbe ענה, celui auquel Yahvé répond est le pauvre עני
} 
la personnification du mal, avec l'expression fréquente "fils de Bélial," avec quelques variantes. ${ }^{40}$

\section{Job}

Dans le Ps 18,21-22: "21 Yahvé me rend selon ma justice, selon la pureté (כבר) (כרי)

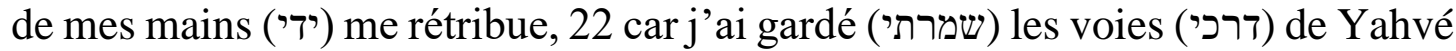
(יהוה) Sans (רשעתי) loillir de mon Dieu", le psalmiste garde les voies de Yahvé, également à la manière de Job, selon Job 23,11ba: “j'ai gardé (שמרתי) ses voies (דרכו)." Mais Job conteste la rétribution, Dieu fait ce qu'il veut, selon Job 23,13: "Mais lui décide, qui le fera changer? Ce qu'il a projeté, il l'accomplit." De même, pour Job, la pureté des mains n'a pas sa rétribution. On relève בר: 2 Sam 22,21.25 ; Ps 18,21.25; Job 9,30 ; 22,30; Is 1,25, pour toute la Bible. La pureté des mains en Job 9,30, est immédiatement contestée avec en 9,31a (Job): "Tu me plonges alors dans l'ordure". Par contre le principe est repris et semble être élargi par Éliphaz en Job 22,30b: “il sera délivré (ונמלט) par la pureté (בבר) (כלפ))

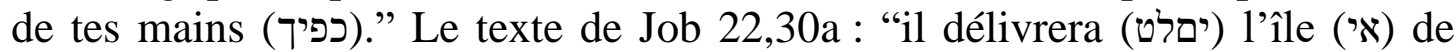
l'innocent (נקי)" délivrera l'homme innocent". ${ }^{42}$ Mais dans ce cas, pourquoi la deuxième partie du verset avec le même verbe מלט? Job 22,30b, semble bien exprimer l'extension des bénéfices de la pureté des mains au-delà du juste lui-même.

En ce qui concerne l'expression ולא-רשעת, de Ps 18,22, le verbe רשע au kal n'apparaît pas par ailleurs dans le Psautier et dans les livres de Samuel sauf donc en 2 Sam 22,22 et Ps 18,22. Par contre dans la continuité des liens avec le livre de Job, il apparaît trois fois dans ce livre, en 9,29; 10,7; 10,15. Là encore la doctrine du Ps 18 est contestée en Job 10,15 : "Suis-je coupable (רשעתי), malheur à moi! suis-je dans mon droit, je n'ose lever la tête, moi saturé d'outrages, ivre de peines!" Dans le livre de Job, tout au moins pour l'opinion qui est présentée comme celle de Job, le sort des uns et des autres ne relève pas de la rétribution.

\section{Sophonie - Zacharie}

D'autres livres présentent une autre opinion plus conforme à la rétribution. La perspective de salut des humbles en Ps 18,28: "toi qui sauves le peuple (עם) des

40 בליעל: 1 Sam 1,16; 2,12; 10,27; 25,17.25; 30,22; 2 S 16,7; 20,1; 22,5; 23,6. En 1 Sam 1,16 il s'agit d'une "fille de Belial". En 1 Sam 2,12, les fils d'Éli sont des "fils de Bélial ", comme les personnages de 1 Sam 10,27. En 1 Sam 25,17, "fils de Belial", désigne Nabal le mari d'Abigayil. En 2 Sam 16,7, Shiméï insulte David "homme de Bélial”. En 2 Sam 20,1 "fils de Bélial”, désigne Shéba, qui rejette la royauté de David. 41 Peut-être une réalité relativement proche, de l'innocent. Plusieurs textes bibliques présentent les "îles" comme un lieu d'espérance, particulièrement dans le livre d'Isaïe, Is 42,4 et 51,5 par exemple. Il y a possiblement un côté exotique.

42 Le texte de la Septante correspond à "il délivre l'homme innocent." La Bible de Jérusalem ajoute une négation à l'innocence: "il délivre même celui qui n'est pas innocent". La polémique existait déjà du temps du livre de Job. 
humbles (עני), et rabaisses les yeux hautains," en réponse à Pr 30,13-14, se trouve confirmée par l'exclusivité de la subsistance du peuple humble en Soph 3,12 : "Je ne laisserai subsister en ton sein, qu'un peuple (עם) humble (עני) et modeste (ודל), et c'est dans le nom de Yahvé que cherchera refuge 13 le reste d'Israël...”. L'humilité est étendue au roi en Za 9,9: "Exulte avec force, fille de Sion! Crie de joie, fille de Jérusalem ! Voici que ton roi (מלכך) vient à toi : il est juste et victorieux, humble (עני), monté (וירכב) (עלכ) sur un âne, sur un ânon, le petit d'une ânesse". Le Ps 18 correspond également à une réaffirmation messianique, en lien avec celle du pauvre et humble, et cela apparaît d'autant plus dans le parallèle de 2 Sam 22, donc dans le cadre des livres de Samuel, en inclusion avec 1 S 2,110. L'application des caractéristiques du pauvre au roi, dans le texte de Zacharie, va donc dans le sens du Ps 18.

\section{Habaquq}

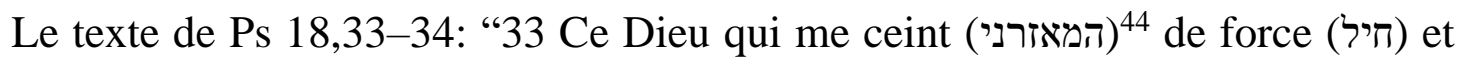

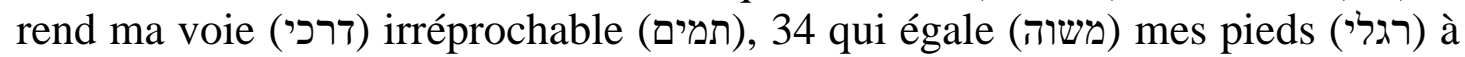
ceux des biches (כאילות) et me tient debout (יעמידני) sur (ועלי) les hauteurs (במתי)," a été repris en Hab 3,1945: "Yahvé mon Seigneur est ma force (חילדי)( mes pieds (רגלי) pareils à ceux des biches (כאילות), sur (ועל) les hauteurs (במותי)

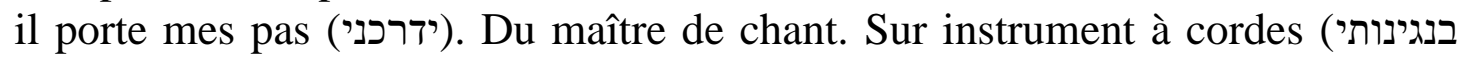
למנצח בנגינות , se retrouve dans les titres de Ps 4,1; 6,1; 54,$1 ; 55,1 ; 67,1 ; 76,1 .{ }^{47}$ Le Ps 18 répond particulièrement aux inquiétudes des titres du deuxième psautier davidique, on peut relever particulièrement ici le Ps 54, où la présence de David est dénoncée à Saül qui veut le tuer.

\section{Ps 68, Jug 5,4}

43 Nous avons relevé qu'en Ps 18,11, Yahvé chevauche (וירכב) un chérubin. L'humilité du roi est soulignée.

44 אזר (verbe): 1 Sam 2,4; 2 Sam 22,40; Ps 18,33.40; 30,12; 65,7; 93,1.

45 Nancy Declaissé-Walford, Rolf A. Jacobson and Beth Laneel Tanner, The Book of Psalms, 192, ils relèvent le lien entre Ps 18,34 (18,33 anglais) et Hab 3,19bc. Ils notent que le Ps 18 doit être compris comme un chant d'action de grâce, non seulement quand les choses vont bien, mais également quand elles vont mal, Hab 3,17-18a.

46 Les livres des prophètes Jonas, Sophonie, Zacharie et Habaquq appartiennent au même rouleau des 12 prophètes, et participent à la réinterprétation de l'activité prophétique. La théophanie de Hab 3,3-15 peut être rapprochée de celle du Ps 18, et nous relevons en Hab 3,13a : "Tu t'es mis en campagne pour sauver (לישע) ton peuple (עמך), pour sauver (לישע) ton oint (את-משיחך)". Nous soulignons l'identification du salut du peuple, au salut du messie. Alison Ruth Gray, Psalm 18, p. 39, rapproche le Ps 18 de Hab 3,3-15 (tendance "archaïsante ").

47 נגינה:Ps 4,1; 6,1; 54,1; 55,1; 61,1 (למנצח על נגינה) 67,1; 69,13; 76,1; 77,7; Hab 3,19; Lam 3,14; 5,14; Job 30,9 pour toute la Bible hébraïque. 


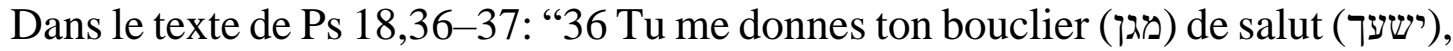

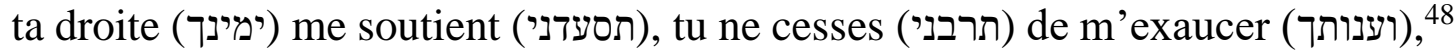
37 tu élargis (תרחיב) mes pas (צעדי) sous moi (תחדי) et mes chevilles (קריבי) n'ont point (מלולו) (מעו)', מגן, fléchi), sont repris les thèmes du bouclier, 18,3.31.36, et du salut, ישע: Ps 18,3.36.47, avec un développement guerrier, qui s'inspire pour une part de l'intervention divine du Ps 68. Ainsi le substantif צעד en Ps 18,37 correspond à l'usage de l'infinitif du verbe en Ps 68,8: "Ô Dieu, quand tu sortis (בצאתך) à la face de ton peuple, quand tu foulas (בצעדך) le désert", Ps 18,36 usant du verbe צעד, plus courant dans le Psautier. L'expression verbale בצעדך, de Ps 68,8, avec infinitif du verbe צער, correspond à celle de Jug 5,4 : "Yahvé, quand tu sortis (בצאתך) de Séir, quand tu t'avanças (בצעדך) des campagnes d'Édom, la terre trembla, les cieux se déversèrent, les nuées fondirent en eau", théophanie reprise par le Ps 68. Cette théophanie a été réinterprétée en Is 34 et 63,1-6, Edom devient la première victime, en faveur du renouveau d'Israël et de Jérusalem. ${ }^{49}$

\section{CONCLUSION}

Le Ps 18 constitue une réaffirmation messianique, de David et sa descendance, qui répond également au livre des Proverbes, au sujet du sort des pauvres exploités au retour de l'exil (Pr 30,1-14), réponse également au sort du pauvre dans le premier psautier davidique qui sur ce point dépend du livre des Proverbes. Pour se faire le Ps 18, s'appuie sur le Ps $68^{50}$ et ses références à $\mathrm{Nb} 10,35$ et Jug 5,4-5, en référence à l'intervention de Yahvé symbolisée par l'arche d'alliance, influence qui d'une manière plus générale concerne l'ensemble du premier psautier davidique Ps 3-41. Dans le cadre de la réponse aux psaumes du second psautier davidique Ps 51-72, et de leurs titres anxiogènes quant au sort de David, le Ps 18 réinterprète les livres de Samuel, en relation à son parallèle de 2 Sam 22, et en inclusion avec le cantique d'Anne de 1 Sam 2,1-10. Le thème du (שאול) Shéol lié aux grandes eaux est commun au livre de Jonas, mais le Ps 18 opère une relecture des livres de Samuel en relisant le terme en fonction du nom de Saül, qui dans les récits des livres de Samuel apparaît comme une menace de mort pour David. Le livre de Job conteste les enseignements de rétribution du Ps 18, tout au moins pour les propos attribués à Job. Éliphaz, par contre semble élargir les bénéfices des mérites du juste à d'autres. Par contre la doctrine du Ps 18 présente des parallèles, reprises et développements chez les petits prophètes,

\footnotetext{
48 Verbe ענה = עני = pépondre, voir = Yahvé répond d'abord aux pauvres.

49 Bernard Gosse, "Le livre d'Isaïe et la vengeance contre Edom, Is 60,1-3 comme réponse à Dt 33,2 (racine זחר), et l'opposition entre les Coréites et les Ezrahites," BN 185 (2020): 24-29: “4. La vengeance contre Edom d'Is 34 comme contrepartie du renouveau de Jérusalem en Is 35 , avec prolongement inversé dans l'oracle contre Edom d'Is 63,1.6 comme contrepartie du renouveau de Jérusalem en Is 60-62".

50 Le Ps 68,6-7, se préoccupe déjà des pauvres, mais ce sont les pauvres classiques, les accidentés de la vie. Le premier psautier davidique concerne les pauvres marginalisés du retour de l'exil.
} 
Sophonie, Zacharie, dans des passages qui relient étroitement les thèmes du roi messianique et du pauvre et Hab 3,19 s'appuie sur Ps 18,33-34.

\section{E BIBLIOGRAPHIE}

Clines, David J. A. The Dictionary of Classical Hebrew, Volume III † - ט. Sheffield: Sheffield Academic Press, 1996.

. The Dictionary of Classical Hebrew, Volume VI $\unlhd$ - 0. Sheffield: Sheffield Phoenix Press, 2007.

Davies, Graham and Robert Gordon. Studies of the Language and Literature of the Bible. Selected Works of J.A. Emerton. SVT 165. Leiden: Brill, 2015.

Declaissé-Walford, Nancy, Rolf A. Jacobson et Beth LaNeel Tanner. The Book of Psalms. New International Commentary on the Old Testament. Grand Rapids: Eerdmans, 2014.

Gosse, Bernard, "La tradition yahviste en Gn 6,5-9,17." HENOCH 15 (1993): 139_ 154.

. 'L'insertion de 2 Sam 22 dans les livres de Samuel, et l'influence en retour sur les titres davidiques du Psautier." Journal of the Ancient Near Eastern Society 27 (2000): 31-47.

. L'influence du livre des Proverbes sur les rédactions bibliques à l'époque Perse. Sup Trans 14. Paris: Gabalda, 2008.

. "Le Ps 19 prolongement du Ps 18: Baal, El, Yahvé, le triple parallélisme synonymique, l'influence du livre des Proverbes et la Loi et David." Zeitschrift für die Alttestamentliche Wissenschaft 129 (2017): 568-582. https://doi.org/10.1515/zaw-2017-4005.

. "The 42 Generations of the Genealogy of Jesus in Matt $1: 1-17$, and the Symbolism of Number 42, Curse or Blessing, in the Bible and in Egypt." Studia Biblica Slovaca 10 (2018): 142-151.

. "2 Samuel 23,1-7, en relation avec le Psautier, le Pentateuque et le livre de Malachie en vue de la réaffirmation de David et sa descendance en réponse à leur substitution par Moïse et en lien avec l'influence du livre des Proverbes sur les rédactions bibliques", Old Testament Essays 31 (2018): 389-398.

- "La réaffirmation messianique du Ps 18 dans la perspective du salut du marginal au retour de l'exil", Zeitschrift für die Alttestamentliche Wissenschaft 130 (2018): 586-601. https://doi.org/10.1515/zaw-2018-4006.

, "La question du pauvre dans le premier psautier davidique, Ps 3-41, comme réponse à Pr 30,13-14.” Old Testament Essays 32 (2019): 544-555.

, "Le livre d'Isaïe et la vengeance contre Edom, Is 60,1-3 comme réponse à Dt 33,2 (racine $z h r$ ), et l'opposition entre les Coréites et les Ezrahites." Biblische Notizen Biblical Notes 185 (2020) : 19-32.

" "L'influence de Pr 30,11-14 sur le Ps 109 dans la continuité de celle de Pr 30,1-14 sur le Ps 18 et la dénonciation de la pratique de la malédiction." 
Zeitschrift für die Alttestamentliche Wissenschaft 132 (2020): 415-426. htpps://doi.org/10.1515/zaw-2020-3003.

Gray, Alison Ruth, Psalm 18 in Words and Pictures. A Reading Through Metaphor. Boston: Brill, 2014.

Kraus, Hans-Johachim, Psalms 1-59: A Commentary. Continental Commentaries. Translated by Hilton C. Oswald. Minneapolis: Augsburg, 1988.

Weber, Beat, Werkbuch Psalmen I. Die Psalmen 1 bis 72. Stuttgart: Verlag w. Kohlhammer, 2001.

Dr Bernard Gosse, Antony, France, Email gosse.bernard.old@orange.fr. ORCID: https://orcid.org/0000-0002-3192-9002. 